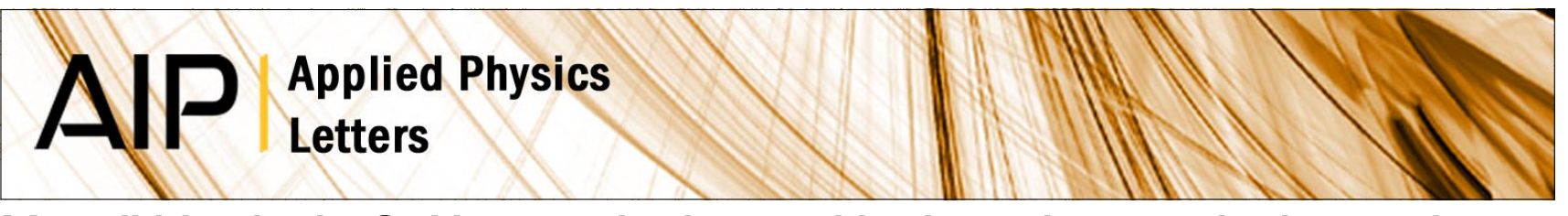

\title{
Monolithic single GaN nanowire laser with photonic crystal microcavity on silicon
}

Junseok Heo, Wei Guo, and Pallab Bhattacharya

Citation: Appl. Phys. Lett. 98, 021110 (2011); doi: 10.1063/1.3540688

View online: http://dx.doi.org/10.1063/1.3540688

View Table of Contents: http://apl.aip.org/resource/1/APPLAB/v98/i2

Published by the AIP Publishing LLC.

Additional information on Appl. Phys. Lett.

Journal Homepage: http://apl.aip.org/

Journal Information: http://apl.aip.org/about/about_the_journal

Top downloads: http://apl.aip.org/features/most_downloaded

Information for Authors: http://apl.aip.org/authors

\section{ADVERTISEMENT}
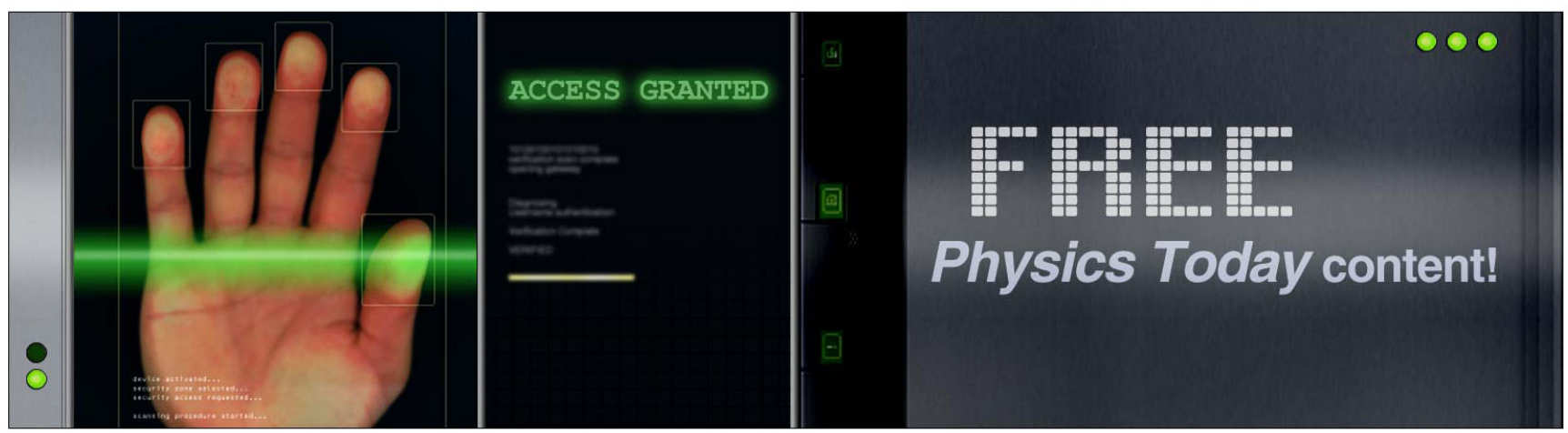


\title{
Monolithic single GaN nanowire laser with photonic crystal microcavity on silicon
}

\author{
Junseok Heo, Wei Guo, and Pallab Bhattacharya ${ }^{\text {a) }}$ \\ Center for Nanoscale Photonics and Spintronics, University of Michigan, Ann Arbor, \\ Michigan 48109-2122, USA
}

(Received 5 November 2010; accepted 22 December 2010; published online 11 January 2011)

\begin{abstract}
Optically pumped lasing at room temperature in a silicon based monolithic single GaN nanowire with a two-dimensional photonic crystal microcavity is demonstrated. Catalyst-free nanowires with low density $\left(\sim 10^{8} \mathrm{~cm}^{-2}\right)$ are grown on $\mathrm{Si}$ by plasma-assisted molecular beam epitaxy. High resolution transmission electron microscopy images reveal that the nanowires are of wurtzite structure and they have no observable defects. A single nanowire laser fabricated on $\mathrm{Si}$ is characterized by a lasing transition at $\lambda=371.3 \mathrm{~nm}$ with a linewidth of $0.55 \mathrm{~nm}$. The threshold is observed at a pump power density of $\sim 120 \mathrm{~kW} / \mathrm{cm}^{2}$ and the spontaneous emission factor $\beta$ is estimated to be 0.08. (C) 2011 American Institute of Physics. [doi:10.1063/1.3540688]
\end{abstract}

There is current interest in the development of nanoscale and ultralow threshold lasers monolithically on silicon for a variety of applications. Several schemes with various degrees of success have been reported. These include both direct epitaxy ${ }^{1,2}$ and wafer bonding schemes. ${ }^{3}$ However, the large mismatch induced defect density and the existence of antiphase domains present serious drawbacks and ultimately limit the long-term reliability of these devices. The successful catalyst-free growth of $\mathrm{Ga}(\mathrm{In}) \mathrm{N}$ nanowires on silicon substrates has been recently demonstrated by a few groups, including ours. ${ }^{4-13}$ The nanowires grow vertically in the wurtzite crystalline form and detailed structural characterizations indicate that they are relatively defect-free. ${ }^{13}$ Consequently, there is much interest in developing nanoscale lasers using semiconductor nanowires. ${ }^{14,15}$ Single nanowires have been shown to function as Fabry-Pérot cavities and waveguides. ${ }^{14,15}$ However, poor reflectivity $(<0.4)($ Ref. 16) between semiconductor and air requires the nanowire to be very long $(\sim 40 \mu \mathrm{m})$ to achieve stimulated emission,,${ }^{16,17}$ which is not conducive to the realization of nanoscale lasers. In this letter we report an optically pumped monolithic single GaN nanowire laser on silicon, with a two-dimensional (2D) photonic crystal (PC) resonant cavity, operating at room temperature.

GaN nanowires were grown on (111) Si substrates in the absence of a foreign metal catalyst in plasma-assisted molecular beam epitaxy (PA-MBE) system. ${ }^{13}$ In order to achieve a low aerial density, the Ga flux and predeposition time of Ga droplets were fixed at $3.5 \times 10^{-8}$ Torr and $1 \mathrm{~min}$, respectively. Figure 1(a) shows an oblique view scanning electron microscopy (SEM) image of GaN nanowires grown on (111) Si with a low density of $\sim 10^{8} \mathrm{~cm}^{-2}$. A single nanowire is vertically aligned and has a length of $\sim 600 \mathrm{~nm}$ and a diameter of $20-50 \mathrm{~nm}$. High-resolution transmission electron microscopy (HRTEM) studies ${ }^{13}$ indicate that the nanowires are relatively defect-free and some evidence of this is provided in the HRTEM image of Fig. 1(b). The diffraction pattern in the inset of Fig. 1(b) reveals that the nanowire has

\footnotetext{
${ }^{\text {a) }}$ Author to whom correspondence should be addressed. Electronic mail: pkb@eecs.umich.edu. Tel.: 734-763-6678. FAX: 734-763-9324.
}

a single crystal wurtzite structure and the $c$-plane is normal to the growth direction.

The wurtzite crystal structure in the GaN nanowire results in an anisotropic dispersion curve near the band edge, with a smaller density of states in the $x$ - and $y$-(in-plane) directions compared to the out-of-plane $z$-direction ( $c$-axis). Therefore, the gain for the same injection is larger for $x$ - and $y$-polarized (TM-polarized) light than for $z$-polarized light. ${ }^{18}$ A 2D photonic crystal with air-holes has a photonic bandgap for TM-polarized light and hence a defect microcavity for TM-polarized light can be easily designed in such a PC. ${ }^{19}$ The device heterostructure, schematically shown in Fig. 2(a), consists of a single $\mathrm{GaN}$ nanowire at the center of a $\mathrm{H} 2$ defect and surrounded by a 2D PC microcavity fabricated in a $\mathrm{TiO}_{2}$ layer and a spin-on-glass (SOG) layer as a low refractive index material to reduce optical loss from the $\mathrm{TiO}_{2}$ layer to the $\mathrm{Si}$ substrate. As a resonator, a $\mathrm{H} 2$ defect PC microcavity is chosen to tolerate any alignment error in a lithography step and is designed by using three-dimensional finite difference time domain (FDTD) simulation. For better estimation, spectroscopic ellipsometry was carried out to characterize the optical properties of $\mathrm{SOG}$ and $\mathrm{TiO}_{2}$. The measured optical refractive indices of SOG and $\mathrm{TiO}_{2}$ at $\lambda$ $=380 \mathrm{~nm}$ are 1.4 and 2.5 , respectively, and the imaginary part of the refractive index of $\mathrm{TiO}_{2}$ is $\sim 0.001$. The $\mathrm{PC}$ is formed with a triangular lattice of air holes of radius $40 \mathrm{~nm}$

(a)
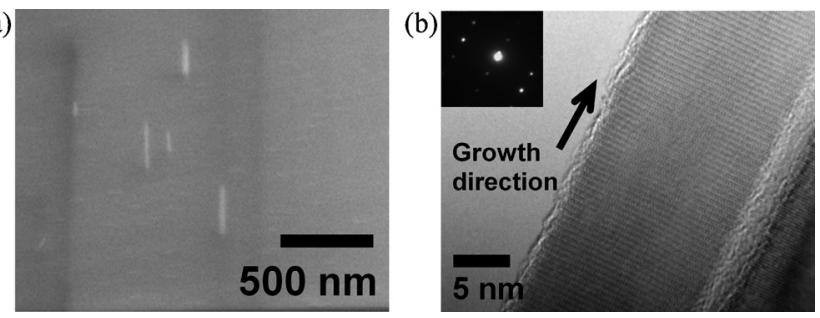

FIG. 1. (a) Cross-sectional SEM images of catalyst-free GaN nanowires grown by PE-MBE with a density of $\sim 1 \times 10^{8} \mathrm{~cm}^{-2}$ on (111) Si. The vertically aligned $\mathrm{GaN}$ nanowires have a length of $\sim 600 \mathrm{~nm}$ and a diameter of 20-50 nm; (b) high resolution TEM image of GaN nanowire with a diameter of $20 \mathrm{~nm}$ which exhibits no observable defect. The inset shows the diffraction pattern indicating the nanowire is a single crystal wurtzite structure with the $c$-axis along the direction of growth. 
(a)
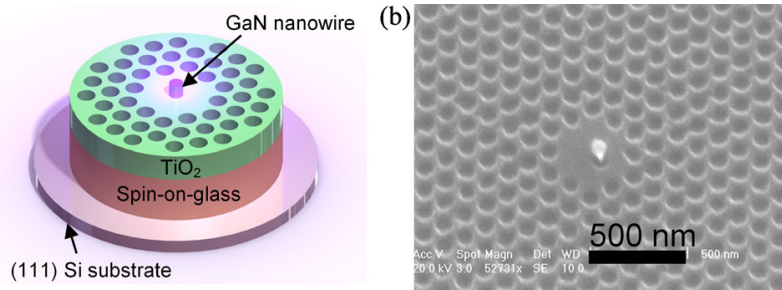

FIG. 2. (Color online) (a) Schematic representation of nanowire laser consisting of a single GaN nanowire and a 2D photonic crystal microcavity; (b) an oblique view SEM image of the fabricated device.

and period $120 \mathrm{~nm}$. A small hollow that is formed around the nanowire in the center of the $\mathrm{H} 2$ defect during device fabrication is assumed to be a partially filled hole with a diameter of $80 \mathrm{~nm}$ in the calculation. The depth of the hollow was roughly estimated to be $\sim 30 \mathrm{~nm}$ based on the amount of $\mathrm{TiO}_{2}$ deposited on top of nanowire. The calculated $Q$-factor, mode volume, and confinement factor $\Gamma$, obtained from the FDTD simulation, are 570, $0.003223 \mu \mathrm{m}^{3}\left[0.92(\lambda / n)^{3}\right]$, and 0.04 , respectively. The modal gain estimated from the $Q$-factor ${ }^{20}$ is $\sim 520 \mathrm{~cm}^{-1}$ and the corresponding optical gain at threshold is $\sim 13000 \mathrm{~cm}^{-1}$, which is achievable in GaN. ${ }^{21}$

Device fabrication is initiated with the spinning of a SOG layer on a low-density nanowire $\left(\sim 10^{8} \mathrm{~cm}^{-2}\right)$ sample grown on (111) Si substrate. The SOG film is baked at 100 and $140{ }^{\circ} \mathrm{C}$ successively for $4 \mathrm{~min}$ to evaporate residual solvents and is then cured at $350{ }^{\circ} \mathrm{C}$ on a hot plate for $30 \mathrm{~min}$ in the absence of oxygen. A SEM image revealed that the sidewalls of the nanowires are not coated by SOG. After the device is cooled down to room temperature, $\mathrm{TiO}_{2}$ is deposited by electron beam evaporation. The thickness of the SOG and $\mathrm{TiO}_{2}$ films, measured by cross-sectional SEM, are 380 and $120 \mathrm{~nm}$, respectively. The 2D PC microcavity is fabricated in the $\mathrm{TiO}_{2}$ layer around the nanowire. Polymethyl methacrylate (PMMA) is spin-coated and a 2D PC, consisting of a triangular lattice of air holes with period $a$ $=120-130 \mathrm{~nm}$ and hole diameter $d=70-80 \mathrm{~nm}$, is pat- terned by electron beam lithography. The pattern in the PMMA layer is transferred to a $\mathrm{TiO}_{2}$ layer by inductively coupled plasma reactive ion etching using $\mathrm{SF}_{6}, \mathrm{C}_{4} \mathrm{~F}_{8}$, and $\mathrm{Ar}$ gases. PMMA is finally removed using oxygen plasma and acetone. Figure 2(b) shows the fabricated device with a single nanowire aligned in the center of the $\mathrm{H} 2$ defect of the $\mathrm{PC}$ microcavity. There is no evidence of $\mathrm{TiO}_{2}$ deposition on the sidewalls of the nanowire.

The device is optically excited at room temperature with a pulsed laser at $\lambda=266 \mathrm{~nm}$ with a pulse duration of $\sim 100$ fs and a repetition rate of $80 \mathrm{MHz}$. The pumping laser is focused on the device with an infinity-corrected objective lens $(\times 27)$ and the emitted light is collected with the same lens. The output spectrum is analyzed with a monochromator (resolution $\sim 0.5 \mathrm{~nm}$ ) and detected with a photomultiplier tube using phase lock-in amplification. Figure 3(a) shows the output spectra recorded for pump power densities of $95 \mathrm{~kW} / \mathrm{cm}^{2}$ (below threshold), $143 \mathrm{~kW} / \mathrm{cm}^{2}$ (slightly above threshold), and $477 \mathrm{~kW} / \mathrm{cm}^{2}$ (above threshold). At low pump power density $\left(\sim 95 \mathrm{~kW} / \mathrm{cm}^{2}\right)$, a broad $\mathrm{GaN}$ bandedge emission with a full width at half maximum of $\sim 10 \mathrm{~nm}$ is observed, which compares well with the reported linewidth of $\sim 12 \mathrm{~nm}$ for GaN bandedge emission. ${ }^{15}$ The output emission also exhibits a fairly narrow peak $(\sim 4.5 \mathrm{~nm})$ at $\lambda=370.4 \mathrm{~nm}$ which is believed to be due to the Purcell effect which enhances spontaneous emission. The enhanced spontaneous emission peak becomes more pronounced near threshold $\left(120 \mathrm{~kW} / \mathrm{cm}^{2}\right)$ and evolves into a coherent lasing peak above threshold. The spectrum recorded at a high pump power density of $477 \mathrm{~kW} / \mathrm{cm}^{2}$ shows a narrow lasing peak at $\lambda=371.3 \mathrm{~nm}$. This peak is made up of two transitions, indicated by the two Gaussian fitting curves, with the dominant transition having a linewidth of $\sim 0.55 \mathrm{~nm}$ limited by the measurement system. The redshift $(\sim 0.9 \mathrm{~nm})$ of the peak wavelength is partly due to bandfilling effects and also due to device heating. The variation of measured emission linewidth with pump power is shown in Fig. 3(b). In order to understand the origin of the dual lasing
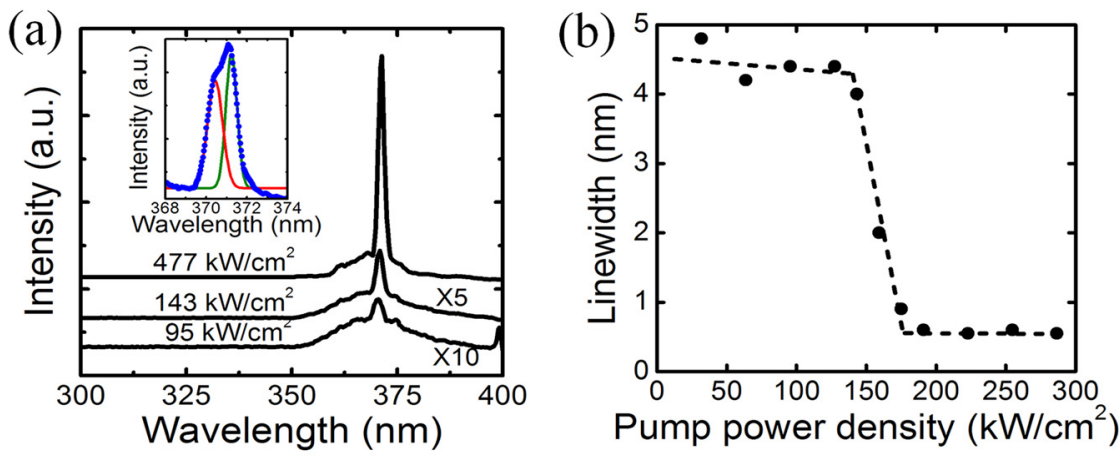

(c)

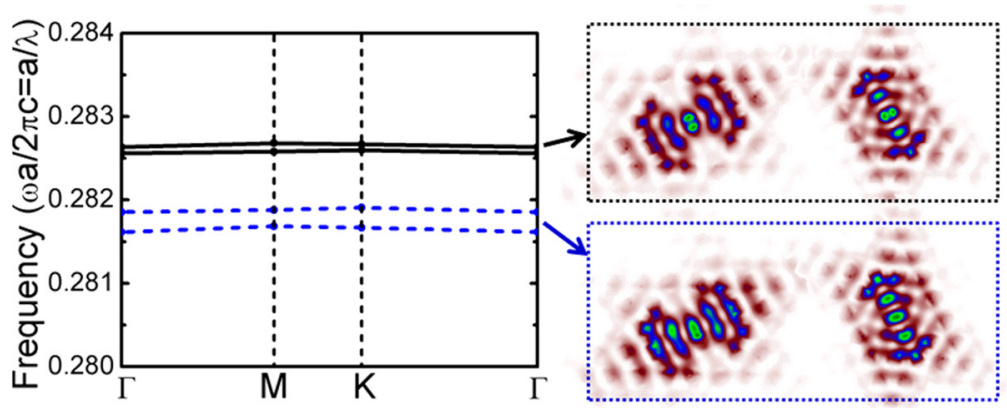

FIG. 3. (Color online) (a) Photoluminescence of the laser at pump power densities of $95 \mathrm{~kW} / \mathrm{cm}^{2}$ (below threshold), $143 \mathrm{~kW} / \mathrm{cm}^{2}$ (near threshold), and $477 \mathrm{~kW} / \mathrm{cm}^{2}$ (above threshold). Spectra are offset for clarity. The inset shows the lasing spectrum (circles), which is matched to the sum of two Gaussian peaks (solid lines); (b) measured linewidth of the dominant peak as a function of the pump power density; (c) calculated resonant modes with the nanowire in the center (solid lines) and off-center by $60 \mathrm{~nm}$ (dashed lines). Mode profiles of each case are shown on the right. 

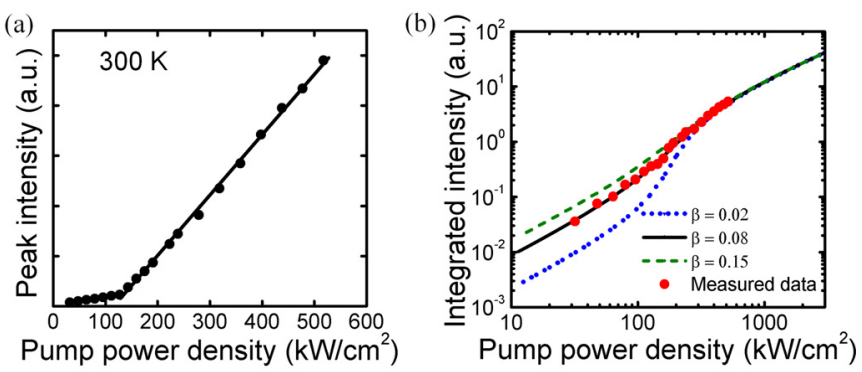

FIG. 4. (Color online) (a) Variation of peak output intensity with pump power (the $L-L$ curve). The change in slope near threshold $\left(\sim 120 \mathrm{~kW} / \mathrm{cm}^{2}\right)$ is clearly observed; (b) log-log plot of spectrally integrated light intensity (circles) vs pump power density. Theoretically calculated light intensity using the rate equations is also shown for spontaneous emission factors $(\beta)$ of $0.02,0.08$, and 0.15 . From a comparison of measured data with the calculated ones, a value of $\beta \sim 0.08$ is estimated.

peaks, the resonant modes were calculated for different positions of the nanowire in the $\mathrm{H} 2$ defect. As shown in Fig. $3(\mathrm{c})$, there are two degenerate modes at $a / \lambda=0.2826$ and 0.28267 (solid lines), which are spectrally very close when the nanowire is placed in the center. As the nanowire is placed off-center in the $\mathrm{H} 2$ defect, the two degenerate modes become more separated, leading to individual modes at $a / \lambda=0.28167$ and 0.28191 (dashed lines). We therefore believe that the dual lasing peaks result from a lifting of the degeneracy of the dominant $\mathrm{H} 2$ cavity modes due to an offcenter positioning of the GaN nanowire within the defect. ${ }^{22}$

The measured variation of the peak intensity of the emitted light with pump power density is shown in Fig. 4(a). A lasing threshold is observed at a pump power density of $\sim 120 \mathrm{~kW} / \mathrm{cm}^{2}$. Calculated plots of emission intensity versus pump power density on a log-log scale are shown in Fig. 4(b). Calculations were done for values of the spontaneous emission factor $\beta$ of $0.02,0.08$, and $0.15{ }^{23}$ The material parameters for $\mathrm{GaN}$ were obtained from Ref. 24. The optical gain was approximated by a linear model, $g(n)=g_{0}\left(n-n_{t r}\right)$, where $g_{0}=2.5 \times 10^{-16} \mathrm{~cm}^{2}$ and $n_{t r}=7.5 \times 10^{18} \mathrm{~cm}^{-3}$. ${ }^{24}$ The measured integrated emission intensity versus pump power density is also plotted alongside the calculated curves. The best agreement between calculated and measured data is obtained for $\beta=0.08$.

In conclusion, we demonstrate the defect-free molecular beam epitaxial growth of low-density GaN nanowires on (111)-oriented silicon substrates. Photonic crystal microcavity lasers were fabricated monolithically with a single nanowire on (111) Si using electron beam lithography and stan- dard dry etching techniques. The steady-state characteristics of these lasers are reported.

The work is supported by the Air Force Office of Scientific Research under Grant No. FA9550-09-1-0634 and the Defense Advanced Research Projects Agency under Grant No. W911NF-07-1-0313. The authors would like to thank Hyun Kum for his help during device fabrication. One of us (J.H.) would like to thank the Samsung Scholarship Program.

${ }^{1}$ J. Yang, Z. Mi, and P. Bhattacharya, J. Lightwave Technol. 25, 1826 (2007).

${ }^{2}$ J. Yang, P. Bhattacharya, and Z. Mi, IEEE Trans. Electron Devices 54, 2849 (2007).

${ }^{3}$ A. W. Fang, H. Park, O. Cohen, R. Jones, M. J. Paniccia, and J. E. Bowers, Opt. Express 14, 9203 (2006).

${ }^{4}$ K. A. Bertness, A. Roshko, L. M. Mansfield, T. E. Harvey, and N. A. Sanford, J. Cryst. Growth 310, 3154 (2008)

${ }^{5}$ O. Landré, R. Songmuang, J. Renard, E. Bellet-Amalric, H. Renevier, and B. Daudin, Appl. Phys. Lett. 93, 183109 (2008)

${ }^{6}$ C. Chèze, L. Geelhaar, O. Brandt, W. Weber, H. Riechert, S. Münch, R. Rothemund, S. Reitzenstein, A. Forchel, T. Kehagias, P. Komninou, G. Dimitrakopulos, and T. Karakostas, Nano Res. 3, 528 (2010).

${ }^{7}$ P. Deb, H. Kim, V. Rawat, M. Oliver, S. Kim, M. Marshall, E. Stach, and T. Sands, Nano Lett. 5, 1847 (2005).

${ }^{8}$ H. Sekiguchi, K. Kishino, and A. Kikuchi, Appl. Phys. Express 1, 124002 (2008).

${ }^{9}$ C. T. Foxon, S. V. Novikov, J. L. Hall, R. P. Campion, D. Cherns, I. Griffiths, and S. Khongphetsak, J. Cryst. Growth 311, 3423 (2009).

${ }^{10}$ R. Calarco, R. J. Meijers, R. K. Debnath, T. Stoica, E. Sutter, and H. Lüth, Nano Lett. 7, 2248 (2007)

${ }^{11}$ H.-W. Lin, Y.-J. Lu, H.-Y. Chen, H.-M. Lee, and S. Gwo, Appl. Phys. Lett. 97, 073101 (2010).

${ }^{12}$ L. Cerutti, J. Ristic, S. Fernandez-Garrido, E. Calleja, A. Trampert, K. H. Ploog, S. Lazic, and J. M. Calleja, Appl. Phys. Lett. 88, 213114 (2006).

${ }^{13}$ W. Guo, M. Zhang, A. Banerjee, and P. Bhattacharya, Nano Lett. 10, 3355 (2010).

${ }^{14}$ J. C. Johnson, H.-J. Choi, K. P. Knutsen, R. D. Schaller, P. Yang, and R. J. Saykally, Nature Mater. 1, 106 (2002).

${ }^{15}$ S. Gradečak, F. Qian, Y. Li, H.-G. Park, and C. M. Lieber, Appl. Phys. Lett. 87, 173111 (2005).

${ }^{16}$ A. V. Maslov and C. Z. Ning, Appl. Phys. Lett. 83, 1237 (2003).

${ }^{17}$ L. Chen and E. Towe, Appl. Phys. Lett. 89, 053125 (2006).

${ }^{18}$ A. V. Maslov and C. Z. Ning, IEEE J. Quantum Electron. 40, 1389 (2004).

${ }^{19}$ R. D. M. John, D. Joannopoulos, and J. N. Winn, Photonic Crystals: Molding the Flow of Light (Princeton University Press, Princeton, 1995).

${ }^{20}$ C. Kim, W. J. Kim, A. Stapleton, J.-R. Cao, J. D. O'Brien, and P. D. Dapkus, J. Opt. Soc. Am. B 19, 1777 (2002).

${ }^{21}$ K. Domen, K. Kondo, A. Kuramata, and T. Tanahashi, Appl. Phys. Lett. 69, 94 (1996).

${ }^{22}$ S.-H. Kim and Y.-H. Lee, IEEE J. Quantum Electron. 39, 1081 (2003).

${ }^{23}$ H.-G. Park, S.-H. Kim, S.-H. Kwon, Y.-G. Ju, J.-K. Yang, J.-H. Baek, S.-B. Kim, and Y.-H. Lee, Science 305, 1444 (2004).

${ }^{24}$ P. Mackowiak and W. Nakwaski, J. Phys. D 31, 2479 (1998). 